\title{
Formulation and Evaluation of Roxithromycin Dispersible Tablets Using Super Disintegrants
}

\author{
Ms.Subhasri Mohapatra ${ }^{1}$, Prof. (Dr.) Sailesh Kumar Ghatuary ${ }^{2}$, \\ Dr. Shradhanjali Patra ${ }^{3}$ \\ ${ }^{1,2}$ Department of Pharmaceutics, Shakti college of Pharmacy, Dulhinpur, Balrampur - 271201 (U.P) \\ ${ }^{3}$ Department of Pharmaceutics,Advance group of Biotechnology\& Paramedical sciences, Kanpur.
}

Abstract: Dispersible tablets of Roxithromycin were prepared using a superdisintegrant such as Primogel powder, Kollidone powder, Crosscaramellose powder, and MCC in different concentration by direct compression method. Formulations were evaluated for the standard of dispersible tablets. It was observed that all the formulations were acceptable with reasonable limits of standard required for dispersible tablets. This study charecterise the most effective superdisintegrant.

Key Words: Dispersible tablet, direct compression, Roxithromycin, super disintegrants.

\section{Introduction:}

Dispersible tablets are uncoated tablets that produce a uniform dispersion or suspension in water at room temperature without stirring. With the increase in the average human life span, drug administration for elderly patients has become more important. Due to decline in swallowing ability with age; a great many elderly patients complain that it is difficult to take medication in the form of tablets. Recently useful dosage form such as rapidly disintegrating or dissolving tablet, have been developed \& applied clinically. The dispersible tablets allow dissolution or dispersion in water prior to administration. Dispersible tablets are easier to administer or swallow than capsules for pediatric, dysphasic patients, mentally ill, unco-operative and nauseated patients, those with conditions of motion sickness, sudden episodes of allergic attack or coughing. Sometimes it may be difficult to swallow conventional products due to unavailability of water. Roxithromycin is a white or, crystalline powder, which is freely soluble in chloroform, acetone,ethanol ,methanol insoluble in water .It has a melting point of $115-125^{\circ} \mathrm{C}$, it posses $\mathrm{pH}$ ranges upto 8-10.

The present investigation was carried out to prepare dispersible tablets of roxithromycin using MCC,primogel(cross $\mathrm{Na}$ starch glycolate,deprogel,explotab),accogel(cross caramellose,cross linked Na $\mathrm{CMC}$ ),kollidone(cross povidone) as asuper disintegrants to establish standards required for the dispersible tablet, to optimize the effective concentration of the disintegrant and to compare the formulations with marketed product.

Materials and Methods: Roxithromycin was provided as a gift sample by Alkem lab.t. ltd. \& Daman, Primogel,kollidone,accogel was provided by cipla lab. ltd.. All the materials used were of standard analytical grade.

\section{Method:}

A) Preparation of Dispersible Tablet [10-12]:

Dispersible tablets of Roxithromycin were prepared using direct compression method after incorporating different disintegrant named as Roxithromycin, Primogel, Kollidone, Cassia Tora, Accga, Mcc, Laclose, Talc, Mag. Stearate, in a concentration 4\%. The composition of formulation is given in Table No 1. The ingredients were thoroughly mixed and passed through sieve no. 22 .

Table1: Formulation of Dispersible Tablet of Norfloxacin

\begin{tabular}{|c|c|c|c|c|c|}
\hline Ingredients & \multicolumn{5}{|c|}{ Formul $^{\mathrm{n}}$ Code } \\
\hline Formul $^{\mathrm{n}}$ Code & $\mathbf{F}_{9}$ & $\mathbf{F}_{10}$ & $\mathbf{F}_{11}$ & $\mathbf{F}_{12}$ & $\mathbf{F}_{13}$ \\
\hline Roxithromycin & 50 & 50 & 50 & 50 & 50 \\
\hline Primogel & 16 & - & - & - & - \\
\hline Kollidone & - & 16 & - & 16 & - \\
\hline Accgogel & - & - & 16 & - & - \\
\hline Mcc & 326 & 244.5 & 244.5 & - & - \\
\hline Lactose & - & 81.5 & 81.5 & 326 & 326 \\
\hline Talc & 4 & 4 & 4 & 4 & 4 \\
\hline Mag. Stearate & 4 & 4 & 4 & 4 & 4 \\
\hline
\end{tabular}


Formulation and evaluation of Roxithromycin Dispersible tablets using Super Disintegrants

\section{B) Evaluation of Formulated Tablet:}

The various formulations were evaluated for hardness, weight variation, friability, disintegration time, Invitro disintegration time, wetting time, uniformity of dispersion, drug content/content uniformity, and dissolution study.

Disintegration time was determined using Thermonic Tablet Disintegration apparatus USP using distilled water as a disintegration medium. Each formulation was tested for uniform dispersion as per official standards. After disintegration beaker was shaken and this fluid was passed through the sieve no.22. Hardness of the tablet was tested Pfizer hardness tester and friability by Roche Friabilator. Drug content was determined by using UV spectrometer (Shimadzu) at $263 \mathrm{~nm}$. Theevaluation parameters shown in Table No 2.

Table2: Evaluations data of formulated dispersible tablet of Roxithromycin

\section{Table 2}

\begin{tabular}{|c|c|c|c|c|}
\hline $\begin{array}{c}\text { Formulation } \\
\text { Code }\end{array}$ & $\begin{array}{c}\text { Thickness } \\
(\mathbf{m m})\end{array}$ & $\begin{array}{c}\text { Av. Wt. } \\
(\mathbf{m g} .)\end{array}$ & $\begin{array}{c}\text { Dissolution } \\
\text { min. })\end{array}$ & $\begin{array}{c}\text { Uniformity of } \\
\text { Dispersion }\end{array}$ \\
\hline $\mathrm{F}_{9}$ & $4.30 \pm 25$ & $387 \pm 2.64$ & $91.80 \pm 1.25$ & \multirow{2}{*}{ Pass } \\
\hline $\mathrm{F}_{10}$ & $4.33 \pm 26$ & $384 \pm 2.13$ & $90 \pm 0.56$ & \multirow{2}{*}{ Through } \\
\hline $\mathrm{F}_{11}$ & $4.33 \pm 27$ & $380 \pm 2.22$ & $90 \pm 0.25$ & \multirow{2}{*}{ \# 20} \\
\hline $\mathrm{F}_{12}$ & $4.35 \pm 29$ & $392 \pm 2.05$ & $95.65 \pm 1.25$ & \\
\hline $\mathrm{F}_{13}$ & $4.38 \pm 0.18$ & $398 \pm 2.69$ & $95.84 \pm 1.35$ & \\
\hline
\end{tabular}

Table 3

\begin{tabular}{|c|c|c|c|c|c|}
\hline $\begin{array}{c}\text { Formulation } \\
\text { Code }\end{array}$ & $\begin{array}{c}\text { Hardness } \\
(\mathbf{k g . / s q . ~ c m})\end{array}$ & $\begin{array}{c}\text { Friability } \\
(\boldsymbol{\%})\end{array}$ & $\begin{array}{c}\text { Disintegration } \\
\text { Test (\%) }\end{array}$ & $\begin{array}{c}\text { Wetting Time } \\
(\mathbf{( s e c} .)\end{array}$ & $\begin{array}{c}\text { Drug Content } \\
(\boldsymbol{\%}) \pm \text { S.D. }\end{array}$ \\
\hline $\mathrm{F}_{9}$ & $4-5$ & 0.70 & 35 & 144 & $92 \pm 0.5$ \\
\hline $\mathrm{F}_{10}$ & $4-5$ & 0.05 & 30 & 143 & $91 \pm 0.35$ \\
\hline $\mathrm{F}_{11}$ & $4-5$ & 0.05 & 30 & 144 & $90 \pm 0.62$ \\
\hline $\mathrm{F}_{12}$ & $2-3$ & 0.95 & 15 & 123 & $94 \pm 0.2$ \\
\hline $\mathrm{F}_{13}$ & $2-3$ & 0.87 & 15 & 125 & $95 \pm 0.2$ \\
\hline
\end{tabular}

\section{C) Dissolution studies:}

Dissolution studies were performed using a dissolution test apparatus USP XXII. (Basket assembly) at $100 \mathrm{rpm}$ using $750 \mathrm{ml}$ of acetate buffer $(\mathrm{pH}-4.0)$ and temperature was maintained at $37+0.5^{\circ}$ through out the study. Ten millimeter of the sample was withdrawn at a regular interval and replaced with an equal volume of phosphate buffer. Samples were filtered and drug content was estimated by UV spectrophotometer at $278 \mathrm{~nm}$. Dissolution data shown in table 4

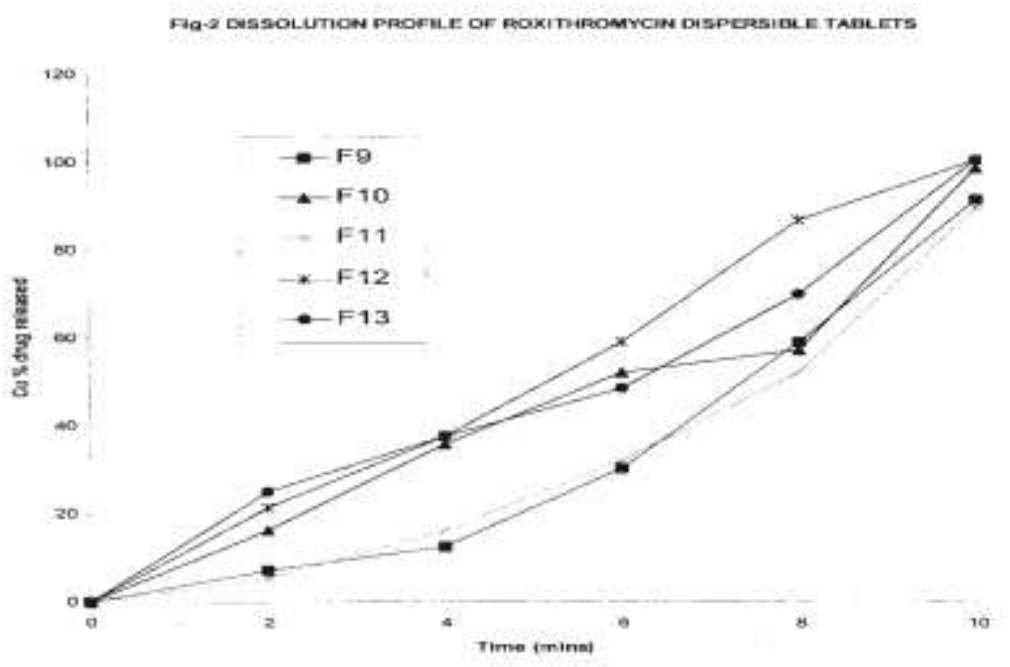


Formulation and evaluation of Roxithromycin Dispersible tablets using Super Disintegrants Table 4

\begin{tabular}{|c|c|c|c|}
\hline Formulation Code & Time (min.) & Dissolution Efficiency & $(\%)$ \\
\hline \multirow{5}{*}{$\mathrm{F}_{9}$} & 2 & $7.20 \pm 0.28$ & \\
\hline & 4 & $12.60 \pm 0.89$ & \\
\hline & 6 & $30.60 \pm 0.58$ & \\
\hline & 8 & $59.40 \pm 0.69$ & \\
\hline & 10 & $91.80 \pm 1.25$ & \\
\hline \multirow{5}{*}{$\mathrm{F}_{10}$} & 2 & $16.52 \pm 0.36$ & \\
\hline & 4 & $36 \pm 0.59$ & \\
\hline & 6 & $52.20 \pm 0.25$ & \\
\hline & 8 & $57.60 \pm 0.58$ & \\
\hline & 10 & $90 \pm 0.56$ & \\
\hline \multirow{5}{*}{$\mathrm{F}_{11}$} & 2 & $5.40 \pm 1.25$ & \\
\hline & 4 & $16.20 \pm 32$ & \\
\hline & 6 & $32.40 \pm 0.87$ & \\
\hline & 8 & $52.20 \pm 0.87$ & \\
\hline & 10 & $90 \pm 0.25$ & \\
\hline \multirow{5}{*}{$\mathrm{F}_{12}$} & 2 & $21.60 \pm 0.58$ & \\
\hline & 4 & $37.8 \pm 0.88$ & \\
\hline & 6 & $81 \pm 0.36$ & \\
\hline & 8 & $59.40 \pm 0.69$ & \\
\hline & 10 & $95.65 \pm 1.25$ & \\
\hline \multirow{5}{*}{$\mathrm{F}_{13}$} & 2 & $25.20 \pm 0.38$ & \\
\hline & 4 & $37.8 \pm 0.12$ & \\
\hline & 6 & $48.60 \pm 1.25$ & \\
\hline & 8 & $70.20 \pm 0.69$ & \\
\hline & 10 & $95.84 \pm 1.35$ & \\
\hline
\end{tabular}

In Vitro Study of Selected Formulated and dispersible Tablet of Roxithromycin (statistical data)

Table -5:

\begin{tabular}{|c|c|c|c|c|c|c|}
\hline $\begin{array}{c}\text { Formulation } \\
\text { Code }\end{array}$ & $\begin{array}{c}\text { Conc. Of } \\
\text { Drug } \\
\text { dissolved }\end{array}$ & $\begin{array}{c}\text { Av. Wt. } \\
\text { (mg) }\end{array}$ & Thickness & $\begin{array}{c}\text { Hardness } \\
\left(\mathrm{Kg} / \mathrm{cm}^{2}\right)\end{array}$ & Friability(\%) & Disinte.Time \\
\hline $\mathrm{F}_{9}$ & $91.80 \pm 1.25$ & $387 \pm 2.64$ & $4.30 \pm 25$ & $4.5 \pm 0.21$ & 0.70 & $35 " \pm 0.11$ \\
\hline $\mathrm{F}_{10}$ & $99 \pm 0.98$ & $384 \pm 2.13$ & $4.33 \pm 26$ & $4.6 \pm 0.17$ & 0.05 & $30 " \pm 0.05$ \\
\hline$F_{11}$ & $90 \pm 0.25$ & $380 \pm 2.22$ & $4.33 \pm 27$ & $4.7 \pm 0.45$ & 0.05 & $30 " \pm 0.04$ \\
\hline $\mathrm{F}_{12}$ & $95.65 \pm 1.25$ & $392 \pm 2.05$ & $4.35 \pm 2.9$ & $4.8 \pm 0.13$ & 0.95 & $15 " \pm 0.09$ \\
\hline $\mathrm{F}_{13}$ & $95.84 \pm 1.35$ & $398 \pm 2.69$ & $4.38 \pm 0.18$ & $4.89 \pm 0.12$ & 0.85 & $15 " \pm 0.01$ \\
\hline
\end{tabular}

Table- 6:

\begin{tabular}{|l|l|l|l|l|}
\hline Formula & \multicolumn{1}{|c|}{$\begin{array}{c}\text { Correl } \\
\text { Coefficient }\end{array}$} & $\begin{array}{c}\text { Slope (Time } v \\
\text { rs \% release }\end{array}$ & T & Rate Const (k) \\
\hline$F_{9}$ & $\mathbf{0 . 9 5 1 4 3}$ & $\mathbf{9 . 0 5 1 4 2}$ & $\mathbf{3 . 1 5}$ & $\mathbf{0 . 2 2}$ \\
\hline$F_{10}$ & $\mathbf{0 . 9 7 6 4 3}$ & $\mathbf{9 . 0 6 3 4 2}$ & $\mathbf{1 . 4 3}$ & $\mathbf{0 . 3 6}$ \\
\hline$F_{11}$ & $\mathbf{0 . 9 7 6 5 1}$ & $\mathbf{8 . 6 6 5 7 1 4}$ & $\mathbf{3 . 4 7}$ & $\mathbf{0 . 2 0}$ \\
\hline$F_{12}$ & $\mathbf{0 . 9 9 7 1 0}$ & $\mathbf{1 0 . 3 1 4 2}$ & $\mathbf{2 . 1 7}$ & $\mathbf{0 . 3 2}$ \\
\hline$F_{13}$ & $\mathbf{0 . 9 8 6 0 2}$ & $\mathbf{9 . 2 8 3 3 7}$ & $\mathbf{2 . 3 9}$ & $\mathbf{0 . 2 9}$ \\
\hline
\end{tabular}

\section{Result and Discussion}

The formulated tablets were evaluated \& taken for statistical analysis study.it shows an efficient formulation for roxithromycin using $4 \%$ cross caramellose which gives hardness $4.89+0.12 \mathrm{~kg} / \mathrm{cm} 2 . \%$ friability $0.85 \& \%$ release within $10 \mathrm{mints}$ was found to be $95.84+1.35$, disintegration time15"+0.01mintswith95+0.02\%drug content.all tablets were formulated with direct compression method.stability studies with this prepared formulation were also performed.the reproducibility of formulation were also checkedby preparing 4 different batches.the \%yeild was found to beuniform with low sd values.anova test was also performed for checking the dissolution test which is indicating reproducibility for f13(roxithromycin with cross caramellose) 
The $\%$ drug content was found to be between $99.00 \%$ to $100.00 \%$, which was within acceptable limits. The hardness was found to be $2.5 \mathrm{Kg} / \mathrm{cm}^{2}$ to $4.0 \mathrm{Kg} / \mathrm{cm}^{2}$, Percent friability was less than $1 \%$ in the entire formulation and values obtained lies between $0.28-0.52$. All the formulations disintegrated between 31-80 seconds The study reveals that formulations prepare shows an efficient formulation d by using $5 \%$ cross caremalloose exhibited good dissolution and uniform dispersion characteristics necessary for dispersion tablets as compared tomarketed, convential tablets of roxithrocin.

\section{Conclusion}

In conclusion, overall result suggests that a 5\% cross caramellose shows better disintegration as compared to marketed tablet.

\section{Acknowledgement}

The author thanks to Alkem laboratory Limited, Daman, , for providing roxithromycin as a gift sample, also thankful to Shri arabindaksha mishra,Assc. Director, Dr. Reddies Lab.,Hydrabad \& Advance group of biotech \& paramedical sciences,kanpur for their coordination \& cooperation .

\section{References}

Handbook of pharmaceutical excipients II edition.

Extra pharmacopoeia by Martin Dale.

Essential of Mediccinal pharmacology K.D. Tripathi $4^{\text {th }}$ ed.

SK Bareja; BM Gupta; Indian J. Pharm., 1968, 30,187.

SK Bareja; BM Gupta; Indian J. Pharm., 1968, 30, 247.

BM Mithal; JL Kasid; Indian J. Pharm., 1964, 26, 316.

BM Mithal; VD Gupta; Indian J. Pharm., 1965, 27, 331

WP Chambers; Quart. J. Pharmacol., 1948, 21, 44.

G Grant; LJ More; J. Sci. Food Ag., 1982, 33, 1324

G Grant; LJ More; JS Stewart; Br. J. Nutr., 1983, 50, 207.

IE Liener, Eds., In; Nutritional significance of lectins in diet, Academic press, Newyork, USA, 1986.

G Grant; Progress food nutrition Sci., 1989, 13, 30.

KPR Chaudhari; R Radha; The Eastern pharmacist, 1998, 163.

KPR Chaudhari; R Rao N; Indian Drugs, 1998, 36, 368.

[15] United states Pharmacopoeia, vol. XXII, USP convention, Rockville, 1990, 1578. 